\title{
Diretrizes da Associação Médica Brasileira para o tratamento do transtorno de ansiedade social
}

\section{Guidelines of the Brazilian Medical Association for the treatment of social anxiety disorder}

\author{
Michelle N. Levitan ${ }^{1,4}$, Marcos H. N. Chagas ${ }^{2,4}$, José A. S. Crippa ${ }^{2,4}$, Gisele G. Manfro ${ }^{3,5}$, Luiz A. B. Hetem², \\ Nathalia C. Andrada ${ }^{6}$, Giovanni A. Salum ${ }^{3,5}$, Luciano Isolan ${ }^{3}$, Maria C. F. Ferrari' ${ }^{2,4}$, Antonio E. Nardi ${ }^{1,4^{*}}$ \\ ${ }^{1}$ Laboratório de Pânico e Respiração. Instituto de Psiquiatria, Universidade Federal do Rio de Janeiro. Rio de Janeiro, Brasil \\ ${ }^{2}$ Departamento de Neurociências e Ciências do Comportamento da Faculdade de Medicina de Ribeirão Preto, Universidade de São Paulo. \\ São Paulo, Brasil \\ ${ }^{3}$ Departamento de Psiquiatria e Medicina Legal. Universidade Federal do Rio Grande do Sul. Rio Grande do Sul, Brasil \\ ${ }^{4}$ INCT Instituto Nacional de Ciência e Tecnologia Translacional em Medicina (CNPq) \\ ${ }^{5}$ INCT Instituto Nacional de Psiquiatria do Desenvolvimento para a Infância e Adolescência (CNPq) \\ ${ }^{6}$ Associação Médica Brasileira
}

\section{Resumo}

Introdução: $\mathrm{O}$ transtorno de ansiedade social (TAS) é o transtorno de ansiedade mais comum, freqüentemente sem remissões, sendo comumente associado com importante prejuízo funcional e psicossocial. A Associação Médica Brasileira (AMB), através do "Projeto Diretrizes", busca desenvolver consensos de diagnóstico e tratamento para as doenças mais comuns. O objetivo deste trabalho é apresentar os achados mais relevantes das diretrizes relativas ao tratamento do TAS, servindo de referência para o médico generalista e especialista. Método: $\mathrm{O}$ método utilizado foi o proposto pela AMB. A busca foi realizada nas bases de dados do MEDLINE (PubMed), Scopus, Web of Science e Lilacs, entre 1980 e 2010. A estratégia utilizada baseou-se em perguntas estruturadas na forma P.I.C.O (acrônimo das iniciais "paciente ou população"; "intervenção, indicador ou exposição"; "controle ou comparação" e; "outcome ou desfecho"). Resultados: Estudos evidenciam que o tratamento farmacológico de primeira linha para adultos e crianças são os inibidores seletivos de recaptação de serotonina e os inibidores de recaptação de serotonina e noradrenalina, enquanto que a terapia cognitivo-comportamental é apontada como melhor tratamento psicoterápico. Além disso, algumas comorbidades psiquiátricas foram associadas a uma pior evolução do TAS. Conclusóes: Apesar da alta prevalência, o TAS acaba por não receber a devida atenção e tratamento. A melhor escolha para o tratamento de adultos é a associação psicoterapia cognitivo-comportamental com inibidores seletivos de recaptação de serotonina e os inibidores de recaptação de serotonina e noradrenalina. Outras opções como benzodiazepínicos ou inibidores da monoaminooxidase devem ser usados como segunda e terceira opção respectivamente.

Descritores: Transtornos fóbicos; Adesão a medicação; Psicoterapia; Diretrizes; Ensaios clínicos controlados aleatórios como assunto

\section{Abstract}

Introduction: Social anxiety disorder (SAD) is the most common anxiety disorder, usually with no remission, and is commonly associated with significant functional and psychosocial impairment. The Brazilian Medical Association (BMA), with the project named Diretrizes (Guidelines, in English), seeks to develop consensus for the diagnosis and treatment of common diseases. The aim of this article is to present the most important findings of the guidelines on the treatment of $S A D$, serving as a reference for the general practitioner and specialist. Method: The method used was proposed by the BMA. The search was conducted in the databases of MEDLINE (PubMed), Scopus, Web of Science and LILACS, between 1980 and 2010. The strategy used was based on structured questions as PICO (acronym formed by the initials of "patient or population", "intervention, display or exhibition", "control or comparison" and "outcome"). Results: Studies show that the firstline pharmacological treatment for adults and children are serotonin selective reuptake inhibitors and serotonin and norepinephrine reuptake inhibitors, whereas cognitive-behavioral therapy is considered the best psychotherapeutic treatment. Moreover, some psychiatric comorbidities were associated with a worse outcome of $S A D$. Conclusions: Despite its high prevalence, SAD does not receive adequate attention and treatment. The best choice for the treatment of adults is a combination of cognitive-behavioral psychotherapy with serotonin selective reuptake inhibitors and serotonin and norepinephrine reuptake inhibitors. Other options as benzodiazepines or monoamine oxidase inhibitors must be used as second and third choices, respectively.

Descriptors: Phobic disorders; Medication adherence; Psychotherapy; Guidelines; Randomized controlled trials as topic
Submetido: 3 de março, 2011

Aceito: 15 de junho, 2011
Correspondência

Antonio E. Nardi

Rua Visconde de Pirajá, 407/702, Rio de Janeiro, RJ

CEP 22410-003

Telefone: (+55 21) 2521-6147 


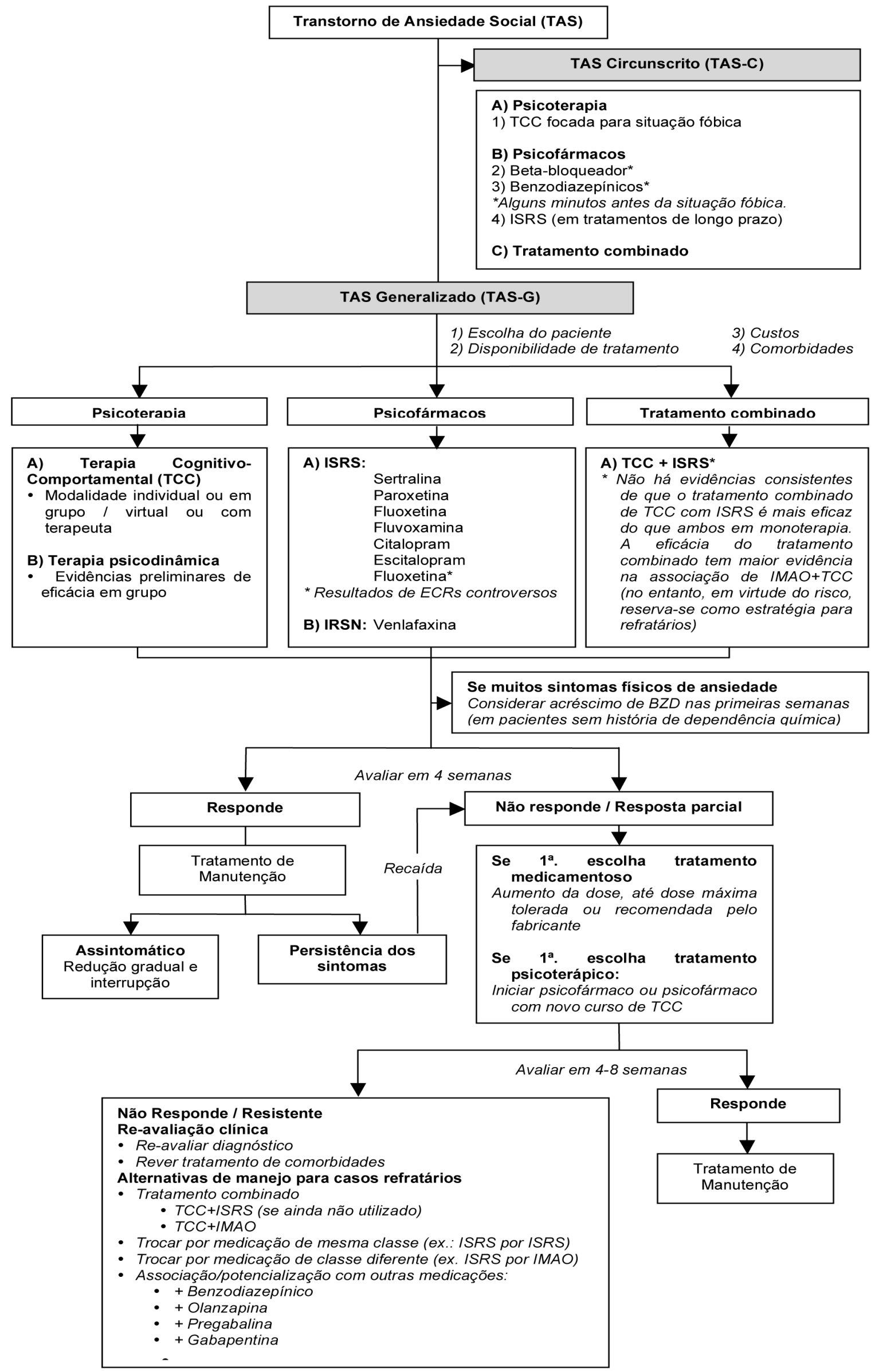




\section{Introdução}

Apesar da alta prevalência e da morbidade significativa do transtorno de ansiedade social (TAS), apenas metade dos pacientes ao longo da vida irão procurar tratamento, com tempo mediano de procura de 16 anos. Além disso, as estimativas americanas apontam para uma adequação mínima de tratamento para essas condições de cerca de $40 \%{ }^{1}$. O tratamento adequado do TAS se inicia com o seu reconhecimento e diferenciação de quadros de timidez (ausência de sofrimento e prejuízo). Posteriormente, é realizada a avaliação das comorbidades comumente associadas ao transtorno, com ênfase em outros transtornos de ansiedade, depressão, abuso de álcool e de outras substâncias psicoativas.

Após o diagnóstico, deve-se proceder para a adequada escolha terapêutica. Nas duas últimas décadas, o crescente reconhecimento do TAS tem sido acompanhado por um maior número de opções de tratamento farmacológico e psicoterápico. A terapia cognitivocomportamental (TCC) e os psicofármacos parecem ter eficácia semelhante em curto prazo $^{2,3}$. A escolha do tratamento deve considerar a disponibilidade do local determinado, a preferência e motivação dos pacientes e os custos associados.

De modo geral, a meta do tratamento do TAS deve ser a remissão total dos sintomas. Apesar das freqüentes dificuldades relacionadas à remissão completa, estudos evidenciam que os sintomas residuais são um dos principais fatores associados à recaída dos quadros de TAS em médio e longo prazo ${ }^{4}$.

Em função da baixa procura por tratamento, pacientes com TAS freqüentemente perdem oportunidades de crescimento pessoal e profissional, desenvolvendo auto-avaliação negativa e isolando-se ainda mais na participação na sociedade. Desta forma, é de extrema importância que profissionais de saúde possam encaminhar ou orientar os pacientes ao melhor tratamento disponível.

A Associação Médica Brasileira (AMB), conjuntamente com o Conselho Federal de Medicina, iniciou no ano de 2000 a elaboração do projeto Diretrizes, com a finalidade de conciliar informações da área médica e padronizar condutas que auxiliem o raciocínio e a tomada de decisão do médico. Em 2009, a AMB convidou a Associação Brasileira de Psiquiatria (ABP) para colaborar com as normas de procedimentos sobre transtornos mentais, baseada em um método que está fundamentado nos conhecimentos da medicina baseada em evidências, através de perguntas com cenário clínico bem definido, estruturadas no acrônimo P.I.C.O. (descrito na seção Método). Assim, os objetivos das presentes diretrizes são: orientar e discutir o tratamento farmacológico e psicoterápico do TAS.

\section{Método}

Estiveram envolvidos neste projeto 10 profissionais de saúde, médicos e psicólogos, membros de grupos de pesquisa produtivos no Brasil e coordenados por uma profissional experiente no método e na realização do projeto. Para desenvolvimento destas diretrizes, foram revisados 80 artigos nas bases de dados do MEDLINE (PubMed), Scopus, Web of Science e Lilacs, entre 1980 e 2010. Além disso, artigos que apresentam relevância na literatura e eram de conhecimento dos autores e manuais diagnósticos (DSM-IV e CID-10) também foram utilizados na elaboração das diretrizes. A estratégia de busca utilizada baseouse em perguntas estruturadas segundo o acrônimo P.I.C.O., formado pelas iniciais de "paciente ou população", "intervenção ou exposição", "controle ou comparação" e "outcome ou desfecho", conforme preconizado pela AMB nesta fase do projeto Diretrizes. A utilização de questões clínicas estruturadas objetivou facilitar a elaboração de estratégias de busca de evidências. Por exemplo, a estratégia de busca para a pergunta "Qual o impacto da depressão no tratamento do TAS?” foi estruturada da seguinte forma: P paciente com TAS e depressão como comorbidade, I - tratamento farmacológico e psicoterapêutico, $\mathrm{C}$ - não há, e $\mathrm{O}$ - impacto da associação. Desta forma, o cruzamento dos seguintes descritores [("phobic disorders" OR "social phobia”) AND "depression" AND ("psychotherapy" OR "drug therapy")] levou aos artigos que passaram pelas seguintes etapas: (I) seleção da evidência; (II) crítica da evidência; (III) extração de resultados; (IV) tradução das pesquisas em grau de recomendação e $(V)$ força da evidência. Estas foram dispostas da seguinte maneira: $A$ : Estudos experimentais ou observacionais de melhor consistência; $B$ : Estudos experimentais ou observacionais de menor consistência; $C$ : Relatos de casos e; $D$ : Opinião desprovida de avaliação crítica, baseada em consensos, estudos fisiológicos ou modelos animais.

Para cruzamentos de acordo com o tema proposto em cada tópico das perguntas P.I.C.O., foram utilizados os descritores: phobic disorders, social phobia, social behavior disorders, mutism, anxiety disorders treatment outcome, behavior therapy, cognitive therapy, psychologic/methods, group psychotherapy, individual psychotherapy, child, adolescent, drug therapy, antidepressive agents, comorbidity, psychotherapy, depression, alcohol drinking, alcohol-related disorders, substance-related disorders, severity of illness index, recurrencelprevention, control, e age of onset. Após a cuidadosa análise deste material, foram selecionados os artigos relativos às perguntas que apresentavam melhor grau de recomendação e força de evidência, os quais originaram e fundamentaram estas diretrizes. Abaixo, estão descritos os achados mais relevantes das diretrizes da $\mathrm{AMB}$ e ABP relativas ao tratamento medicamentoso e psicoterápico do TAS.

\section{O tratamento psicoterápico é eficaz para o TAS? Quais as técnicas mais recomendadas?}

Estudos mostram que a farmacoterapia tende a provocar resultados um pouco melhores do que a TCC, porém o tratamento combinado se mostra superior às monoterapias. Em um estudo 
controlado randomizado (ECR), o percentual de pacientes com TAS em remissão foi de $46,9 \%$ no tratamento combinado e $7,4 \%$ para o placebo $(\mathrm{OR}=11,03$, IC 95\%; 2,23-54,57). Na monoterapia, as taxas de remissão foram de $22.1 \%$ no grupo com fenelzina $(\mathrm{OR}=3.70$; IC 95\%; 0,82-19,14) e 8.8\% no grupo de TCC $(1.21 ; 0,19-7,81)^{5}$ (A).

As técnicas utilizadas na psicoterapia cognitivo-comportamental incluem psicoeducação, relaxamento muscular progressivo, treinamento de habilidades sociais, exposição imaginária e ao vivo, vídeo feedback e reestruturação $\operatorname{cognitiva}^{6}$ (D). Embora haja controvérsia a respeito de qual elemento da psicoterapia cognitivo-comportamental é mais eficaz, sabese que trabalhar o componente cognitivo é essencial, com $84 \%$ dos pacientes apresentando melhora dos sintomas e persistência dos resultados no seguimento de um ano ${ }^{7}$ (A). Quando confrontados com uma situação social, pacientes com TAS superestimam as conseqüências negativas do contato social e desvalorizam suas habilidades sociais a serem utilizadas na situação exposta. Intervenções psicoterapêuticas buscam a identificação e reestruturação das cognições distorcidas, além de utilizarem técnicas comportamentais de exposição gradativa às situações sociais e treinamento de habilidades sociais para melhora do desempenho social ${ }^{8}(\mathbf{D})$.

Há preditores de abandono do tratamento com TCC antes do início da mesma, que devem ser reconhecidos para indicação mais precisa do tratamento: nível mais alto de sintomas do TAS, associação com comorbidades como transtornos de depressão e personalidade esquiva e abandono escolar ${ }^{9}$ (A).

2. A terapia em grupo mostra-se mais adequada no tratamento do TAS do que a terapia individual?

A TCC em grupo para o TAS é uma escolha bastante utilizada, embora poucos estudos comparativos com a terapia individual tenham sido conduzidos. Na TCC em grupo, há a facilidade do uso da exposição às situações temidas, uma vez que o grupo é utilizado como rede de suporte para os desafios terapêuticos. Existe a possibilidade de se desenvolver habilidades e comportamentos por observação dos outros participantes ${ }^{10}(\mathbf{B})$.

O uso da terapia em grupo apresenta algumas limitaçôes, como o longo período de espera para a formação do grupo e uma menor flexibilidade de agendamento de sessóes, o que pode levar a uma maior taxa de abandono em comparação à terapia individual ${ }^{11}(\mathbf{B})$. Além disso, é possível que questôes individuais deixem de ser trabalhadas ou que o grupo faça uma esquiva generalizada a algumas situaçôes ${ }^{12}(\mathbf{B})$. Dois estudos apontam para uma baixa taxa de melhora do TAS com a TCC em grupo, variando de $38^{13}$ (B) a $60 \%{ }^{14}($ A).

Ao contrário do que vem sendo apontado como o melhor preditor de resultados no TAS, um estudo randomizado com duração de quatro meses evidenciou que metade dos participantes com TAS tratados com TCC em grupo ainda apresentavam diagnóstico de TAS, contra 13,6\% da terapia individual. Além disso, a TCC individual produziu melhora dos sintomas avaliados por meio de escalas referentes à gravidade do TAS de $84 \%$ versus $44 \%$ do grupo $^{10}(\mathbf{B})$. A TCC individual parece facilitar o acesso às crenças disfuncionais $\mathrm{e}$ comportamentos de segurança, além de fornecer um ambiente menos amedrontador para o paciente. Porém, apesar da sua maior eficácia, deve-se considerar o contexto de aplicação, no qual realizar uma terapia em grupo pode significar um custo mais baixo e maior número de pacientes atendidos.

\section{Qual o tratamento psicoterápico do TAS em crianças e adolescentes?}

Estudos evidenciam que o tratamento psicoterápico mais eficaz com crianças e adolescentes com TAS é o cognitivocomportamental. Ao comparar vários tipos de TCC para o tratamento de crianças com TAS, não houve diferenças significativas entre tratamento individual ou em grupo, e o envolvimento dos pais foi eficaz nas duas formas de terapia ${ }^{15}$ (B).

Em uma metanálise ${ }^{16}$ (A) que avaliou oito tratamentos cognitivo-comportamentais em crianças, todos encontraram uma diminuição significativa no nível de prejuízo associado ao TAS nos participantes ao final do tratamento, com tamanhos de efeito variando entre 4,06 $\pm 1,31^{17}$ (B) e 1,25 $\pm 0,24^{18}$ (B). Além disso, três relataram tamanhos do efeito moderados a grandes no aumento da competência social, variando entre $0,91 \pm 0,22^{19}$ (B) até $0,59 \pm 0,10^{18}(\mathbf{B})$. Os sintomas do TAS também foram reduzidos nestes tratamentos.

Devido ao importante papel que os pais de crianças com TAS exercem sobre o quadro, como solucionar os problemas pelos filhos e reforçar a esquiva social, alguns estudos demonstraram que o envolvimento dos familiares no tratamento produz melhores resultados $^{15,18}$ (A).

Em estudos somente com adolescentes, a TCC mostrou-se mais eficaz no tratamento dos sintomas do TAS e de comorbidades freqüentes como a depressão quando comparada à terapia de suporte educacional-familiar ${ }^{20}(\mathbf{B})$. $\mathrm{O}$ tratamento psicológico que combina treinamento em habilidades sociais, reestruturação cognitiva e exposição gradativa é apontado como o mais eficaz para crianças, adolescentes e adultos.

\section{Qual o tratamento medicamentoso indicado para - TAS em adultos?}

Duas classes de psicofármacos são consideradas de primeira linha no tratamento farmacológico do TAS, tanto pelo fato de terem se mostrados eficazes em vários ECRs com placebo, quanto pela segurança de seus efeitos adversos. Essas drogas de escolha são os inibidores seletivos de recaptação de serotonina (ISRS) e os inibidores de recaptação de serotonina e noradrenalina (IRSN) ${ }^{21}$ (A). Apesar dos benzodiazepínicos (BZD) também apresentarem forte evidência de eficácia, não são considerados medicações de primeira linha em função do perfil pouco favorável de efeitos adversos e risco de abuso e dependência ${ }^{22}$ (D), assim como 
os antidepressivos inibidores da monoaminoxidade (IMAO), devido ao aumento do risco de crise hipertensiva e acidente vascular encefálico quando as recomendações dietéticas não são estritamente cumpridas pelo paciente. Os antidepressivos tricíclicos, além de não demonstrarem tanta eficácia, podem agravar os sintomas fóbicos ansiosos, principalmente devido aos efeitos colaterais como tremores finos e sudorese ${ }^{23,24}$ (B) (Figura 1).

\section{Inibidores seletivos de recaptação de serotonina (ISRS)}

Entre os ISRS, o escitalopram, a fluvoxamina, a sertralina e a paroxetina são consideradas medicações de primeira linha para o tratamento do $\operatorname{TAS}^{21}$ (A). Embora seja muito utilizada na prática clínica, apenas um estudo mostrou maior eficácia da fluoxetina em relação ao placebo na população $\operatorname{adulta}^{3}$ (A). Outros dois estudos falharam em demonstrar essa superioridade ${ }^{25,26}$ (A). Apesar da eficácia do citalopram só ter sido testada em um $\mathrm{ECR}^{27}(\mathrm{~A})$, parece não haver diferenças em relação à eficácia entre as medicaçôes consideradas de primeira linha ${ }^{21}(\mathbf{A})$.

O uso de sertralina com doses flexíveis entre 50 e $200 \mathrm{mg} /$ dia mostrou-se eficaz em reduzir os sintomas fóbicos ansiosos após 12 semanas, com taxa de resposta de $55,6 \%$ versus $29 \%$ de resposta com o uso de placebo ${ }^{28}(\mathbf{A})$, com número necessário para tratar (NNT) de 3. Um estudo prévio de 20 semanas apontou para os mesmos resultados, com taxa de resposta de $53 \%$ versus $29 \%$ do grupo placebo, com NNT de $4^{29}$ (A).

Da mesma forma, o escitalopram mostrou-se superior ao placebo em doses de 5 a $20 \mathrm{mg} /$ dia em um seguimento de 12 e 24 semanas $^{30}(\mathbf{A})$. A resposta à dosagem do escitalopram entre $10 \mathrm{e}$ $20 \mathrm{mg}$ também foi superior ao placebo, com taxas de $54 \%$ contra $39 \%$ e NNT de $7^{31}$ (A).

Em um estudo duplo cego randomizado de 12 semanas, a paroxetina com dosagem máxima de $50 \mathrm{mg} / \mathrm{dia}$ melhorou sintomas fóbico-sociais $(65,7 \%$ vs. $32,4 \%, \mathrm{NNT}=3)$ em relação ao placebo $^{32}$ (A). Em outro estudo com o mesmo desenho experimental, $55 \%$ dos pacientes que usaram paroxetina e $23,9 \%$ dos pacientes em uso de placebo apresentaram resposta ao tratamento de acordo com a escala Clinical Global Impression (CGI), com NNT de $3^{33}$ (A). Estes resultados foram replicados por outros estudos $32,34,35$ (A).

De forma semelhante, em um estudo duplo-cego controlado por placebo com a fluvoxamina (dose média de $202 \mathrm{mg} / \mathrm{dia}$ ), 42,9\% do grupo com TAS que recebia medicação e 22,7\% do grupo placebo apresentaram melhora significativa dos sintomas do TAS com NNT de $5^{36}(\mathbf{A})$. Outros estudos ratificam estes achados, nos quais a fluvoxamina demonstrou-se eficaz no tratamento do TAS em doses entre 150 e $300 \mathrm{mg} / \mathrm{dia}^{36-38}(\mathbf{A})$.

\section{Inibidores de recaptação de serotonina e nor- adrenalina (IRSN)}

A venlafaxina de liberação estendida foi avaliada no tratamento do TAS em um estudo de 28 semanas. Quando comparada ao placebo, o grupo com a droga ativa apresentou taxas de resposta ( $58 \%$ vs. $33 \%, \mathrm{NNT}=4)$ e remissão ( $31 \%$ vs. $16 \%, \mathrm{NNT}=7)$ superiores, tanto em baixas doses $(75 \mathrm{mg} / \mathrm{dia})$ como em altas doses $(150 \mathrm{mg} \text { e } 225 \mathrm{mg} / \mathrm{dia})^{39}$ (A). Outros estudos encontraram resultados similiares ${ }^{40-42}(\mathbf{A})$, apontando que a venfalaxina é uma medicação eficaz, segura e bem tolerada no tratamento do TAS.

\section{Inibidores de monoamino-oxidase (IMAO)}

Apesar de eficazes, os IMAO impõem restrições alimentares importantes em virtude do risco de interação com a tiramina presente em certos alimentos, podendo provocar aumento repentino na pressão sanguínea e reações hipertensivas ${ }^{43}(\mathbf{B})$. Desta forma, deve haver cautela em seu uso, além de medidas informativas a serem adotadas pelo médico, como o fornecimento de uma lista de alimentos e medicaçôes que devem ser evitados.

Em particular, a fenelzina, um IMAO irreversível, demonstrouse bastante eficaz no tratamento do $\operatorname{TAS}^{44}(\mathbf{B})$. Em ensaios clínicos, os IMAO, juntamente com BZD e ISRS, se mostraram superiores no tratamento do TAS, porém a maior evidência de tolerabilidade e eficácia foi associada aos $\operatorname{ISRS}^{45}$ (A).

\section{Benzodiazepínicos (BZD)}

Os BZD (clonazepam, bromazepam, alprazolam) são usados freqüentemente no tratamento dos transtornos ansiosos. $\mathrm{O}$ clonazepam, em doses entre 0,5 e $3 \mathrm{mg} / \mathrm{dia}$, mostrou-se efetivo no tratamento do TAS com taxas de resposta de $78,3 \%$ contra $20 \%$ no grupo placebo. Em geral, o clonazepam foi bem tolerado, porém o grupo em uso do mesmo apresentou mais tonturas e instabilidade postural que o grupo placebo ${ }^{46}(\mathbf{A})$.

Da mesma forma, o bromazepam mostrou-se efetivo em um estudo de 12 semanas $^{47}(\mathbf{A})$. O único ensaio clínico que avaliou o alprazolam falhou em mostrar eficácia desta medicação ${ }^{2}(\mathbf{A})$. O risco de abuso e dependência contra-indica o uso de BZD em pacientes com história de dependência e coloca esse tipo de medicação como uma opção de segunda linha no tratamento do TAS.

\section{Beta-bloqueadores}

Embora muito utilizados na prática clínica, os beta-bloqueadores (atenolol, pindolol, propranolol) não apresentam evidência de superioridade no tratamento do TAS em relação ao placebo. A recomendação de seu uso limita-se a situações de desempenho que não fazem parte da rotina do indivíduo, visando reduzir sintomas somáticos associados à ansiedade de desempenho ${ }^{48}(\mathbf{A})$.

\section{Qual o tratamento medicamentoso do TAS em crianças e adolescentes?}

Infelizmente, pouca atenção tem sido dada ao uso de medicações para o TAS em crianças e adolescentes. Alguns estudos abertos com ISRS têm mostrado resultados promissores, apontando-os como medicaçôes de primeira linha para o tratamento do TAS nesta população.

Em um estudo de 16 semanas com crianças e adolescentes de 8 a 17 anos com dosagem média de paroxetina de $25 \mathrm{mg} / \mathrm{dia}$, 77,6 
\% do grupo com TAS respondeu à medicação, com NNT de 3, contra 38,3\% do grupo placebo ${ }^{49}$ (A).

A fluoxetina $(20 \mathrm{mg} / \mathrm{dia})$ utilizada por pacientes entre 7 e 17 anos mostrou-se superior ao placebo no tratamento dos sintomas do TAS em um ensaio clínico de 12 semanas, com melhora de $61 \%$ vs. $35 \%$ dos casos, respectivamente, fornecendo um NNT de $4^{50}$ (A).

Em um estudo aberto de 8 semanas com sertralina (dose média de $123 \mathrm{mg} / \mathrm{dia}$ ), $36 \%$ das crianças com TAS foram classificadas como responsivas ao tratamento e $29 \%$ como parcialmente responsivas. Além disso, houve uma diminuição do desconforto em tarefas sociais e nos escores das escalas associadas à timidez e ansiedade ${ }^{51}$ (B).

O escitalopram, utilizado por 12 semanas $(10-20 \mathrm{mg} / \mathrm{dia})$, provocou melhora dos sintomas do TAS e na qualidade de vida em $65 \%$ dos participantes, com tamanhos de efeito variando de 0,9 a $1,9^{52}(\mathbf{B})$.

Em outro estudo, adolescentes com TAS apresentaram remissão de sintomas em 52,9\% com a TCC, $48,6 \%$ com fenelzina e $78,1 \%$ com tratamento combinado (psicoterapia e farmacoterapia) em comparação com adultos com TAS, os quais obtiveram melhora de 23,5\% com TCC, 25,7\% para fenelzina e 53,1\% para o tratamento combinado, com diferença significativa para o tratamento combinado 5 (A).

\section{Qual o período mínimo de manutenção do tratamento medicamentoso em pacientes com TAS?}

Após a remissão dos sintomas do TAS, a manutenção do tratamento medicamentoso diminui a possibilidade de recaídas ${ }^{53,54}$ (A). Um estudo com a paroxetina em dosagem média de 36,67 mg/ dia demonstrou que o tratamento de manutenção por um período de 24 semanas diminui a taxa de recaídas significativamente, de forma que apenas $14 \%$ dos pacientes que mantinham o uso da paroxetina apresentaram recaídas, contra 39\% do grupo em uso de placebo, com OR=0,24 (IC 95\%; 0,14-0,43; p<0,001) ${ }^{54}$ (A).

Um estudo semelhante com o escitalopram em dosagens flexíveis de $10-20 \mathrm{mg} / \mathrm{dia}$ apresentou resultados similares. Os pacientes que continuaram o uso do antidepressivo por um período de manutenção de 24 semanas apresentaram uma taxa de recaídas significativamente menor do que o grupo controle em uso de placebo, o qual apresentou um risco de recaída 2,8 vezes mais alto do que o grupo tratado com escitalopram ${ }^{53}$ (A).

Um período mínimo de 24 semanas após remissão dos sintomas fóbico-sociais tem sido considerado adequado para evitar possíveis recaídas $^{53,54}(\mathbf{A})$, porém períodos mais prolongados podem ser necessários.

\section{Há diferenças no tratamento do TAS circunscrito e generalizado?}

Das poucas pesquisas que avaliaram as diferenças nos resultados do tratamento psicoterápico em pacientes com os subtipos de TAS, todos foram realizados com TCC em grupo. Em um estudo, o subtipo circunscrito respondeu significativamente melhor a TCC do que o subtipo generalizado (79\% vs. $47 \%)$, embora os dois grupos tenham apresentado melhora ${ }^{55}(\mathbf{B})$. Já em outra investigação os subtipos não diferiram quanto à melhora geral, porém o subtipo circunscrito terminou a terapia com um melhor índice de funcionamento (grau de comprometimento pelo TAS) ${ }^{56}(\mathbf{B})$.

Os estudos farmacológicos são em sua grande maioria conduzidos com pacientes com o TAS de subtipo generalizado, uma vez que selecionar pacientes com apenas uma limitação social que procurem os serviços de saúde não é comum. Em uma revisão da literatura, a partir da avaliação de três ensaios duplo-cegos de 12 semanas controlados com paroxetina (20-50 mg/dia), não foram encontradas diferenças nas respostas ao tratamento entre o grupo circunscrito e generalizado ${ }^{57}$ (A). A fluvoxamina também se mostrou eficaz para os dois tipos de TAS com dosagem média de $150 \mathrm{mg} /$ dia em um estudo duplo cego de $12 \operatorname{semanas}^{37}$ (A).

No TAS circunscrito, para o tratamento da ansiedade de desempenho, freqüentemente são recomendados os beta-bloqueadores e BZD, que melhoram a sintomatologia fisiológica do TAS no momento da exposição, mas não em longo prazo. Faz-se importante, porém, lembrar que estas medicações não tratam as comorbidades freqüentemente associadas ao TAS e devem ser usadas com cautela pelo risco de dependência ${ }^{58}$ (A).

\section{Há diferenças entre a resposta terapêutica do TAS de início precoce e de início tardio?}

Poucos estudos abordam a influência da idade de início do TAS na resposta terapêutica. Um estudo brasileiro tratou pacientes com TAS de início precoce ( $<18$ anos) e de início tardio ( $\geq 18$ anos) com ISRS, tricíclicos e BZD por 10 semanas, e não encontrou diferenças na melhora de sintomas do TAS entre os grupos ${ }^{59}$. De acordo com outros estudos realizados, a idade de início do TAS não parece ser um preditor de resposta ao tratamento.

\section{0 que é o mutismo seletivo?}

O mutismo seletivo caracteriza-se por uma falha da criança em falar quando seria esperado que ela se comunicasse, a qual não é explicada por desentendimento do idioma ou não-compreensão do que está sendo falado. Geralmente a recusa não ocorre em toda exposição social, mas com relação a algumas situações ou pessoas $^{60}(\mathbf{C})$. Apesar de ainda não haver consenso sobre sua classificação, é considerado por grande parte dos autores como uma forma mais grave e precoce do TAS, ocorrendo normalmente entre os 5 e 11 anos de idade, com uma distribuição entre 0,8 e $1,9 \%{ }^{61}(\mathbf{B})$.

Observações clínicas indicam que pais de crianças com mutismo seletivo reforçam o comportamento da criança ao apoiarem quando os filhos não respondem aos outros. Além disso, alguns estudos evidenciam que parte das crianças com este quadro apresentam comportamentos desafiadores e agressivos ${ }^{62}(\mathbf{C})$. As crianças são frequentemente definidas pelos pais como tímidas e socialmente evitativas ${ }^{63}(\mathbf{C})$.

Para inclusão de pacientes com mutismo seletivo, os estudos utilizam os critérios do DSM-IV (APA), avaliados por professores e pais, com duração de pelo menos um mês e interferência no 
desempenho social e escolar da criança ${ }^{64}(\mathbf{B})$. Uma boa avaliação para mutismo seletivo é feita através de entrevistas com pessoas do convívio da criança, como professores, babá e familiares.

\section{Qual o tratamento do mutismo seletivo?}

Em função da baixa prevalência do mutismo seletivo, poucos estudos abordaram seu tratamento. No maior estudo aberto realizado com fluoxetina, durante 9 semanas com 21 crianças com mutismo seletivo, utilizou-se uma dosagem média de $28,1 \mathrm{mg} / \mathrm{dia}$ e máxima de $60 \mathrm{mg} /$ dia nas últimas semanas do tratamento, onde nenhuma criança obteve melhora com dosagem inferior a $20 \mathrm{mg} /$ dia. Ao final do tratamento, $76 \%$ preencheram critérios de melhora do quadro, com uma modificação da média de interferência dos sintomas de 47,1 (moderada interferência) para 67,5 (pouca interferência; $\mathrm{p}<0,001)$, sendo inversamente proporcional à resposta ao tratamento ${ }^{65}(\mathbf{C})$.

Em um estudo recente, 17 crianças com mutismo seletivo participaram de um programa para melhora dos sintomas em um hospital infantil ${ }^{66}(\mathbf{C})$. Oito crianças tomaram uma solução líquida de fluoxetina e duas de sertralina com dosagem máxima de 10 a 25mg/ dia e de 25 a 50mg/dia, respectivamente. Sete crianças não obtiveram autorização para a administração de medicação e não participaram do restante do estudo. Após seis meses, as crianças medicadas mostraram melhora em escalas referentes ao mutismo seletivo superior àquelas sem medicação, embora ainda com diagnóstico.

No âmbito psicológico, um estudo de pequeno porte envolvendo cinco crianças e pais durante oito semanas foi realizado no formato de TCC em grupo. Os pais receberam sessōes com informaçōes psicoeducativas e intervenções necessárias para aumentar a confiança do filho em se expor. O objetivo era a diminuição da ansiedade nas exposiçōes e o aumento do repertório de comunicação. Todas as crianças obtiveram melhora no grau de confiança ao falar na escola e diminuição da ansiedade. Duas crianças não preencheram mais o diagnóstico de mutismo seletivo ${ }^{67}(\mathbf{C})$.

11. Qual o impacto da comorbidade com depressão no tratamento de pacientes com TAS?

Embora esteja bem estabelecido que as comorbidades aumentam a gravidade e prejuízo associado do TAS, o impacto da depressão no tratamento dos pacientes com TAS é pouco explorado na literatura. Em um estudo que avaliou a eficácia da fluoxetina e TCC no tratamento do TAS, evidenciou-se que a presença de sintomas depressivos em pacientes com TAS foi associada a um quadro mais grave de TAS e pior evolução do tratamento ao longo do tempo. Além disso, pacientes que abandonaram o tratamento apresentavam uma maior taxa de sintomas depressivos ${ }^{68}(\mathbf{A})$.

Estudos que investigaram o papel da depressão na resposta dos pacientes com TAS a TCC demonstram que esta comorbidade parece não afetar a resposta terapêutica em curto prazo ${ }^{69,70}(\mathbf{B})$. Em um desses estudos, no entanto, pacientes com depressão demonstraram ser mais propensos a recair dos sintomas de TAS no seguimento, sugerindo a necessidade de uma intervenção adicional para manutenção dos ganhos da TCC em pacientes com depressão ${ }^{69}(\mathbf{B})$. A TCC para depressão em pacientes com comorbidade com TAS parece apresentar resultados satisfatórios para ambas as condiçôes ${ }^{71}$ (A).

12. Qual o impacto da comorbidade com abuso e dependência de álcool e drogas ilícitas no tratamento de pacientes com TAS?

Pacientes com TAS apresentam aumentado risco de comorbidade com dependência de álcool ${ }^{72}$ (A), assim como uma alta prevalência de uso de álcool e drogas ilícitas ${ }^{73}$ (D). O uso de álcool é associado à maior freqüência de comorbidades como transtornos de humor e de personalidade, enquanto que o uso concomitante de álcool e drogas ilícitas aumenta ainda mais a probabilidade de incidência de outras comorbidades psiquiátricas e está associado a pensamentos de morte e tentativas de suicídio ${ }^{73}$ (D). Pacientes com TAS são mais propensos a usar álcool em situações de desafeto, pressão ou censura $\operatorname{social}^{74}(\mathbf{A})$, com prevalência estimada deste uso em $3,3 \%{ }^{73}(\mathbf{D})$

Pacientes com TAS e dependência de álcool apresentam prejuízos funcionais em diversos domínios, incluindo perda do suporte social, menor satisfação nas relações interpessoais e maior tendência à manifestação de doenças físicas. No TAS comórbido com álcool e drogas, há uma maior busca por profissionais de saúde e a uma maior utilização de medicação para controle dos sintomas. Assim, a necessidade de uma maior assistência mental e física em pacientes com TAS e dependência de álcool resulta em grandes custos pessoais e sociais ${ }^{74}$ (A). A presença de TAS não aumentou o risco de abuso de álcool $(\mathrm{OR}=1,23$, IC 95\%, 0,97-1,57, p=0,09), mas aumentou significativamente a chance de dependência $(\mathrm{OR}=2,26$, IC $95 \%, 1,88-2,70, \mathrm{p}<0,001)^{74}(\mathbf{A})$.

\section{Qual o impacto da comorbidade com outros transtornos de ansiedade no tratamento de pacien- tes com TAS?}

Estudos mostram que o tratamento psicoterápico do TAS comórbido a outros transtornos de ansiedade apresenta uma taxa similar de sucesso quando comparado ao TAS puro, todavia, poucos estudos farmacológicos avaliaram esta associação. Em um estudo com TCC de 12 semanas com adultos, grupos com TAS puro e TAS associado com transtorno de ansiedade generalizada exibiram uma percentagem de melhora dos sintomas de ansiedade similar, inclusive durante o seguimento ${ }^{75}(\mathbf{B})$.

Em um estudo com TCC em crianças, os transtornos de ansiedade comórbidos não foram associados com o desfecho da terapia, onde $68,4 \%$ dos pacientes sem comorbidades e $70,6 \%$ dos pacientes com comorbidades tiveram remissão do TAS. Os pacientes que permaneceram com as comorbidades após o tratamento apresentavam menor susceptibilidade a remissão dos sintomas do $\operatorname{TAS}^{76}(\mathbf{B})$. 
Em um estudo farmacológico, pacientes com TAS e transtorno de pânico (TP) comórbido receberam tranilcipromina, um IMAO, durante 12 semanas, com doses de $30 \mathrm{mg} /$ dia ou $60 \mathrm{mg} /$ dia. Não foram encontradas diferenças significativas na redução de ataques de pânico. O grupo que recebeu $30 \mathrm{mg} /$ dia teve os ataques de pânico reduzidos em $69,6 \%$, enquanto o grupo de $60 \mathrm{mg} /$ dia teve redução de $74,8 \%$, porém, a dose maior mostrou-se mais eficaz na diminuição de sintomas de ansiedade social $(30 \mathrm{mg} / \mathrm{dia}=-17,9 \pm 14,7$ e $60 \mathrm{mg} / \mathrm{dia}=-35,0 \pm 14,8)$. Desta forma, apesar da dosagem maior ter sido necessária para a melhora do TAS, o TP comórbido não pareceu afetar o desfecho do tratamento farmacológico do $\operatorname{TAS}^{77}$ (A).

\section{Conclusões}

A partir da extensa revisão da literatura realizada, os tratamentos que mais se destacaram para o TAS foram os ISRS, IRSN e a TCC. Além da eficácia dos ISRS encontrada nos ECR, o menor número de efeitos colaterais e a melhor tolerância dos pacientes colocam-nos como opção medicamentosa de primeira linha no tratamento do TAS. A TCC também foi associada à boa adesão dos pacientes com TAS, além de alcançar resultados semelhantes ou superiores aos dos fármacos.

O projeto Diretrizes, da $A M B$ em parceria com a $A B P$, visa facilitar e auxiliar nas decisões dos médicos em geral, devendo apresentar clareza, aplicabilidade clínica e relevância prática. Apesar da alta prevalência, o TAS é freqüientemente associado à personalidade, e acaba por não receber a devida atenção e tratamento.

As diretrizes sobre diagnóstico e diagnóstico diferencial do TAS já foram realizadas ${ }^{78}$, disponibilizando um guia para avaliação correta do transtorno, que agora é complementado pelos algoritmos de tratamento. As vantagens e desvantagens dos tratamentos são expostas, de forma que o profissional possa discutir com seu paciente sobre a melhor escolha de tratamento. Percebese, no entanto, que a maior parte dos estudos medicamentosos foi realizada há mais de duas décadas e há a necessidade de novas pesquisas. Além disso, estudos com crianças ainda são escassos, inviabilizando o tratamento precoce e um melhor prognóstico na idade adulta.

Conclui-se que a apresentação destas diretrizes permitirá que profissionais de saúde possam facilmente identificar as opçóes terapêuticas disponíveis para crianças e adultos com TAS e selecionar o tratamento mais adequado ao seu paciente, provendo informações envolvidas nesta escolha e manejando com segurança os resultados. 
Financiamento e conflito de interesses

\begin{tabular}{|c|c|c|c|c|c|c|c|}
\hline $\begin{array}{l}\text { Membro do } \\
\text { grupo de } \\
\text { autores }\end{array}$ & $\begin{array}{l}\text { Local de } \\
\text { trabalho }\end{array}$ & $\begin{array}{c}\text { Verba de } \\
\text { pesquis } \\
\text { a }\end{array}$ & $\begin{array}{c}\text { Outro } \\
\text { apoio à } \\
\text { pesquisa } \\
\text { ou } \\
\text { educação } \\
\text { médica } \\
\text { continuad } \\
\text { a }\end{array}$ & $\begin{array}{c}\text { Honorários de } \\
\text { palestrante }\end{array}$ & $\begin{array}{l}\text { Participaçã } \\
\text { o acionária }\end{array}$ & $\begin{array}{l}\text { Consultor/Consel } \\
\text { ho consultivo }\end{array}$ & Outro $^{3}$ \\
\hline Levitan MN & UFRJ & $\mathrm{CNPq}$ & - & - & - & - & - \\
\hline Chagas MHN & USP-RP & - & - & - & - & - & - \\
\hline Crippa JAS & USP-RP & $\mathrm{CNPq}^{* *}$ & - & - & - & - & $\begin{array}{c}\text { Czec } \\
\text { Neurolo } \\
\text { gical } \\
\text { Foundat } \\
\text { ion } \\
\text { Aché } \\
\text { Elly Lilly } \\
\text { Roche }\end{array}$ \\
\hline Manfro GG & UFRGS & $\begin{array}{l}\text { CNPq }^{*} \\
\text { FIPE- } \\
\text { HCPA }^{*}\end{array}$ & - & - & - & - & Roche* \\
\hline Hetem LAB & USP-RP & - & - & - & - & - & - \\
\hline Andrada NC & AMB & - & - & - & - & - & - \\
\hline Salum GA & UFRGS & CAPES & - & - & - & - & - \\
\hline Isolan L & UFRGS & - & - & - & - & - & - \\
\hline Ferrari MCF & USP-RP & FAPESP & - & - & - & - & - \\
\hline Nardi AE & UFRJ & $\mathrm{CNPq}^{* \star}$ & - & $\begin{array}{c}\text { Glaxo-SmithKline* } \\
\text { Roche }^{*}\end{array}$ & - & $\begin{array}{l}\text { Aché }^{*} \\
\text { CNPq }^{*}\end{array}$ & $\begin{array}{l}\text { Editora } \\
\text { Artmed }^{*}\end{array}$ \\
\hline
\end{tabular}

* Modesto

** Significativa

*** Significativa. Montantes fornecidos à instituição do autor ou a colega para pesquisa onde o autor tem participação, não diretamente ao autor.

\section{Referências}

1. Wang PS, Lane M, Olfson M, Pincus HA, Wells KB, Kessler RC. Twelve-month use of mental health services in the United States: results from the National Comorbidity Survey Replication. Arch Gen Psychiatry. 2005;62(6):629-40.

2. Gelernter CS, Uhde TW, Cimbolic P, Arnkoff DB, Vittone BJ, Tancer ME, Bartko JJ. Cognitive-behavioral and pharmacological treatments of social phobia.A controlled study. Arch Gen Psychiatry. 1991;48(10):938-45.

3. Davidson JR, Foa EB, Huppert JD, Keefe FJ, Franklin ME, Compton JS, Zhao N, Connor KM, Lynch TR, Gadde KM. Fluoxetine, comprehensive cognitive behavioral therapy, and placebo in generalized social phobia. Arch Gen Psychiatry. 2004;61(10):1005-1013.

4. Fava GA, Grandi S, Rafanelli C, Ruini C, Conti S, Belluardo P. Long-term outcome of social phobia treated by exposure. Psychol Med. 2001;31(5):899905.

5. Blanco C, Heimberg RG, Schneier FR, Fresco DM, Chen H, Turk CL, Vermes D, Erwin BA, Schmidt AB, Juster HR, Campeas R, Liebowitz MR. A placebo-controlled trial of phenelzine, cognitive behavioral group therapy, and their combination for social anxiety disorder. Arch Gen Psychiatry. 2010;67(3):286-95.

6. Huppert JD, Roth DA, Foa EB. Cognitive-behavioral treatment of social phobia: new advances. Curr Psychiatry Rep. 2003; 5 (4):289-96.

7. Clark DM, Ehlers A, Hackmann A, McManus F, Fennell M, Grey N, Waddington L, Wild J. Cognitive therapy versus exposure and applied relaxation in social phobia: A randomized controlled trial. J Consult Clin Psychol. 2006;74 (3):568-78.

8. Rapee RM, Heimberg RG. A cognitive-behavioral model of anxiety in social phobia. Behav Res Ther. 1997;35(8):741-56.

9. Eskildsen A, Hougaard E, Rosenberg NK. Pre-treatment patient variables as predictors of drop-out and treatment outcome in cognitive behavioural therapy for social phobia: A systematic review. Nord J Psychiatry. 2010;64(2):94-105. 10. Stangier U, Heidenreich T, Peitz M, Lauterbach W, Clark DM. Cognitive therapy for social phobia: individual versus group treatment. Behav Res Ther. 2003;41(9):991-1007.

11. Scholing A, Emmelkamp PM. Exposure with and without cognitive therapy for generalized social phobia: effects of individual and group treatment. Behav Res Ther. 1993;31(7):667-81.

12. Wlazlo Z, Schroeder-Hartwig K, Hand I, Kaiser G, Munchau N. Exposure in vivo vs social skills training for social phobia: long-term outcome and differential effects. Behav Res Ther. 1990;28(3):181-93.

13. Mattick RP, Peters L. Treatment of severe social phobia: effects of guided exposure with and without cognitive restructuring. J Consult Clin Psychol. 1988;56(2):251-60.

14. Heimberg RG, Liebowitz MR, Hope DA, Schneier FR, Holt CS, Welkowitz LA, Juster HR, Campeas R, Bruch MA, Cloitre M, Fallon B, Klein DF. Cognitive behavioral group therapy vs phenelzine therapy for social phobia: 12-week outcome. Arch Gen Psychiatry. 1998;55 (12): 1133-141. 
15. Silverman WK, Pina AA, Viswesvaran C. Evidence-based psychosocial treatments for phobic and anxiety disorders in children and adolescents. $J$ Clin Child Adolesc Psychol. 2008; 37(1):105-30.

16. Segool NK, Carlson JS. Efficacy of cognitive-behavioral and pharmacological treatments for children with social anxiety. Depress Anxiety. 2008;25(7):620-31.

17. Albano AM, Marten PA, Holt CS, Heimberg RG, Barlow DH. Cognitivebehavioral group treatment for social phobia in adolescents.A preliminary study. J Nerv Ment Dis. 1995;183 (10):649-656.

18. Spence SH, Donovan C, Brechman-Toussaint M. The treatment of childhood social phobia: the effectiveness of a social skills training-based, cognitive-behavioural intervention, with and without parental involvement. J Child Psychol Psychiatry. 2000;41(6):713-26.

19. Gallagher HM, Rabian BA, McCloskey MS. A brief group cognitivebehavioral intervention for social phobia in childhood. J Anxiety Disord. 2004;18(4):459-79.

20. Silverman WK, Kurtines WM, Ginsburg GS, Weems CF, Rabian B Serafini LT. Contingency management, self-control, and education support in the treatment of childhood phobic disorders: a randomized clinical trial. J Consult Clin Psychol. 1999;67(5):675-87.

21. Ipser JC, Kariuki CM, Stein DJ. Pharmacotherapy for social anxiety disorder: a systematic review. Expert Rev Neurother. 2008;8(2):235-57.

22. Lydiard RB. The role of drug therapy in social phobia.J Affect Disord. 1998;50 Suppl 1:S35-39

23. Simpson HB, Schneier FR, Campeas RB, Marshall RD, Fallon BA, Davies $S$, Klein DF, Liebowitz MR. Imipramine in the treatment of social phobia. J Clin Psychopharmacol. 1998;18 (2):132-35.

24. Versiani M, Mundim FD, Nardi AE, Liebowitz MR. Tranylcypromine in social phobia. J Clin Psychopharmacol. 1988;8(4):279-83.

25. Clark DM, Ehlers A, McManus F, Hackmann A, Fennell M, Campbel H, Flower T, Davenport C, Louis B. Cognitive therapy versus fluoxetine in generalized social phobia: a randomized placebo-controlled trial. J Consult Clin Psychol. 2003;71(6):1058-1067.

26. Kobak KA, Greist JH, Jefferson JW, Katzelnick DJ. Fluoxetine in social phobia: a double-blind, placebo-controlled pilot study. J Clin Psychopharmacol. 2002;22(3):257-62.

27. Furmark T, Appel L, Michelgard A, Wahlstedt K, Ahs F, Zancan S, Jacobsson E, Flyckt K, Grohp M, Bergstrom M, Pich EM, Nilsson LG, Ban M, Langstrom B, Fredrikson M. Cerebral blood flow changes after treatment of social phobia with the neurokinin-1 antagonist GR205171, citalopram, or placebo. Biol Psychiatry. 2005;58(2):132-42.

28.Liebowitz MR, DeMartinis NA, Weihs K, Londborg PD, Smith WT, Chung H, Fayyad R, Clary CM. Efficacy of sertraline in severe generalized social anxiety disorder: results of a double-blind, placebo-controlled study. $J$ Clin Psychiatry. 2003;64(7):785-92.

29. Van Ameringen MA, Lane RM, Walker JR, Bowen RC, Chokka PR, Goldner EM, Johnston DG, Lavallee YJ, Nandy S, Pecknold JC, Hadrava V, Swinson RP. Sertraline treatment of generalized social phobia: a 20-week, double-blind, placebo-controlled study. Am J Psychiatry. 2001;158 (2):275-81.

30. Lader M, Stender K, Burger V, Nil R. Efficacy and tolerability of escitalopram in 12- and 24-week treatment of social anxiety disorder: randomised, double-blind, placebo-controlled, fixed-dose study. Depress Anxiety. 2004;19 (4):241-48

31. Kasper S, Stein DJ, Loft H, Nil R. Escitalopram in the treatment of socia anxiety disorder: randomised, placebo-controlled, flexible-dosage study. $\mathrm{Br} J$ Psychiatry. 2005;186:222-26.

32. Baldwin D, Bobes J, Stein DJ, Scharwachter I, Faure M. Paroxetine in social phobia/social anxiety disorder. Randomised, double-blind, placebocontrolled study. Paroxetine Study Group. Br J Psychiatry. 1999;175:120-26. 33. Stein MB, Liebowitz MR, Lydiard RB, Pitts CD, Bushnell W, Gergel I. Paroxetine treatment of generalized social phobia (social anxiety disorder): a randomized controlled trial. JAMA. 1998;280 (8):708-13.

34. Stein DJ, Berk M, Els C, Emsley RA, Gittelson L, Wilson D, Oakes $\mathrm{R}$, Hunter B. A double-blind placebo-controlled trial of paroxetine in the management of social phobia (social anxiety disorder) in South Africa. $S$ Afr Med J. 1999;89(4):402-6.

35. Allgulander C. Paroxetine in social anxiety disorder: a randomized placebocontrolled study. Acta Psychiatr Scand. 1999;100 (3):193-8.

36. Stein MB, Fyer AJ, Davidson JR, Pollack MH, Wiita B. Fluvoxamine treatment of social phobia (social anxiety disorder): a double-blind, placebocontrolled study. Am J Psychiatry. 1999;156 (5):756-60.

37. van Vliet IM, den Boer JA, Westenberg HG. Psychopharmacological treatment of social phobia; a double blind placebo controlled study with fluvoxamine. Psychopharmacology (Berl). 1994;115(1-2):128-34.

38. Asakura S, Tajima O, Koyama T. Fluvoxamine treatment of generalized social anxiety disorder in Japan: a randomized double-blind, placebocontrolled study. Int J Neuropsychopharmacol. 2007;10 (2):263-74.

39. Stein MB, Pollack MH, Bystritsky A, Kelsey JE, Mangano RM. Efficacy of low and higher dose extended-release venlafaxine in generalized social anxiety disorder: a 6-month randomized controlled trial. Psychopharmacology (Berl). 2005;177 (3):280-88.

40. Rickels K, Mangano R, Khan A. A double-blind, placebo-controlled study of a flexible dose of venlafaxine ER in adult outpatients with generalized social anxiety disorder. J Clin Psychopharmacol. 2004;24(5):488-96.

41. Liebowitz MR, Mangano RM, Bradwejn J, Asnis G. A randomized controlled trial of venlafaxine extended release in generalized social anxiety disorder. J Clin Psychiatry. 2005;66 (2):238-47

42. Allgulander C, Mangano R, Zhang J, Dahl AA, Lepola U, Sjodin I, Emilien G. Efficacy of Venlafaxine ER in patients with social anxiety disorder: a double-blind, placebo-controlled, parallel-group comparison with paroxetine. Hum Psychopharmacol. 2004;19(6):387-96.

43. Schneier FR, Blanco C, Campeas R, Lewis-Fernandez R, Lin SH, Marshall R, Schmidt AB, Sanchez-Lacay JA, Simpson HB, Liebowitz MR. Citalopram treatment of social anxiety disorder with comorbid major depression. Depress Anxiety. 2003;17(4):191-96.

44. Versiani M, Nardi AE, Mundim FD, Alves AB, Liebowitz MR, Amrein R. Pharmacotherapy of social phobia. A controlled study with moclobemide and phenelzine. Br J Psychiatry 1992; 161:353-60.

45. Blanco C, Schneier FR, Schmidt A, Blanco-Jerez CR, Marshall RD, Sanchez-Lacay A, Liebowitz MR. Pharmacological treatment of social anxiety disorder: a meta-analysis. Depress Anxiety. 2003;18(1):29-40.

46. Davidson JR, Potts N, Richichi E, Krishnan R, Ford SM, Smith R, Wilson WH. Treatment of social phobia with clonazepam and placebo. $J$ Clin Psychopharmacol. 1993;13(6):423-28.

47. Versiani M, Nardi A, Figuera I, Marques C. Double-blind placebo controlled trial with bromazepam in social phobia. J Bras Psiquiatr. 1997;46:167-71.

48. James I, Savage I. Beneficial effect of nadolol on anxiety-induced disturbances of performance in musicians: a comparison with diazepam and placebo. Am Heart J.1984;108(4 Pt 2):1150-1155.

49. Wagner KD, Berard R, Stein MB, Wetherhold E, Carpenter DJ, Perera P, Gee M, Davy K, Machin A. A multicenter, randomized, double-blind, placebo-controlled trial of paroxetine in children and adolescents with social anxiety disorder. Arch Gen Psychiatry. 2004;61(11):1153-1162.

50. Birmaher B, Axelson DA, Monk K, Kalas C, Clark DB, Ehmann M, Bridge J, Heo J, Brent DA. Fluoxetine for the treatment of childhood anxiety disorders. J Am Acad Child Adolesc Psychiatry. 2003; 42 (4):415-23.

51. Compton SN, Grant PJ, Chrisman AK, Gammon PJ, Brown VL, March JS. Sertraline in children and adolescents with social anxiety disorder: an open trial. J Am Acad Child Adolesc Psychiatry. 2001;40(5):564-71.

52. Isolan L, Pheula G, Salum GA Jr, Oswald S, Rohde LA, Manfro GG. An open-label trial of escitalopram in children and adolescents with social anxiety disorder. J Child Adolesc Psychopharmacol. 2007;17(6): 751-60.

53. Montgomery SA, Nil R, Durr-Pal N, Loft H, Boulenger JP. A 24-week randomized, double-blind, placebo-controlled study of escitalopram for the prevention of generalized social anxiety disorder. J Clin Psychiatry. 2005;66(10):1270-1278. 
54. Stein DJ, Versiani M, Hair T, Kumar R. Efficacy of paroxetine for relapse prevention in social anxiety disorder: a 24-week study. Arch Gen Psychiatry. 2002;59(12):1111-1118.

55. Brown EJ, Heimberg RG, Juster HR. Social phobia subtype and avoidant personality disorder: Effect on severity of social phobia, impairment, and outcome of cognitive behavioral treatment. Behav Ther. 1995;26:467-86.

56. Turner SM, Beidel DC, Wolff PL, Spaulding S, Jacob RG. Clinical features affecting treatment outcome in social phobia. Behav Res Ther. 1996;34(10): 795-804.

57. Stein MB, Sareen J, Hami S, Chao J. Pindolol potentiation of paroxetine for generalized social phobia: a double-blind, placebo-controlled, crossover study. Am J Psychiatry. 2001;158 (10):1725-1727.

58. Davidson JR. Pharmacotherapy of social phobia. Acta Psychiatr Scand Suppl. 2003; (417):65-71.

59. Menezes GB, Fontenelle LF, Versiani M. Early-onset social anxiety disorder in adults: clinical and therapeutic features. Rev Bras Psiquiatr. 2005;27(1):32-6.

60. Black B, Uhde TW. Psychiatric characteristics of children with selective mutism: a pilot study. J Am Acad Child Adolesc Psychiatry. 1995;34(7):847-56.

61. Bergman RL, Piacentini J, McCracken JT. Prevalence and description of selective mutism in a school-based sample. J Am Acad Child Adolesc Psychiatry. 2002;41(8):938-46.

62. Steinhausen HC, Juzi C. Elective mutism: an analysis of 100 cases. J Am Acad Child Adolesc Psychiatry. 1996;35(5):606-14.

63. Ford MA, Sladeczek IE, Carlson J, Kratochwill TR. Selective mutism: Phenomenological characteristics. Sch Psychol. 1998;13(3):192-27.

64. Kumpulainen K, Rasanen E, Raaska H, Somppi V. Selective mutism among second-graders in elementary school. Eur Child Adolesc Psychiatry. 1998;7(1):24-29.

65. Dummit ES, 3rd, Klein RG, Tancer NK, Asche B, Martin J. Fluoxetine treatment of children with selective mutism: an open trial. J Am Acad Child Adolesc Psychiatry. 1996;35(5):615-21.

66. Manassis K, Tannock R. Comparing interventions for selective mutism: a pilot study. Can J Psychiatry. 2008;53(10):700-3.

67. Sharkey L, Mc Nicholas F, Barry E, Begley M, Ahern S. Group therapy for selective mutism - a parents' and children's treatment group. J Behav Ther Exp Psychiatry. 2008;39(4):538-45.

68. Ledley DR, Huppert JD, Foa EB, Davidson JR, Keefe FJ, Potts NL. Impact of depressive symptoms on the treatment of generalized social anxiety disorder. Depress Anxiety. 2005;22(4):161-67.

69. Marom S, Gilboa-Schechtman E, Aderka IM, Weizman A, Hermesh H. Impact of depression on treatment effectiveness and gains maintenance in social phobia: a naturalistic study of cognitive behavior group therapy. Depress Anxiety. 2009;26(3):289-300.

70. Erwin BA, Heimberg RG, Juster H, Mindlin M. Comorbid anxiety and mood disorders among persons with social anxiety disorder. Behav Res Ther. 2002;40(1):19-35.

71. Smits JA, Minhajuddin A, Jarrett RB. Cognitive therapy for depressed adults with comorbid social phobia. J Affect Disord. 2009;114(1-3): 271-78.

72. Buckner JD, Schmidt NB. Understanding social anxiety as a risk for alcohol use disorders: fear of scrutiny, not social interaction fears, prospectively predicts alcohol use disorders. J Psychiatr Res. 2009;43(4):477-83.

73. Robinson J, Sareen J, Cox BJ, Bolton J. Self-medication of anxiety disorders with alcohol and drugs: Results from a nationally representative sample. J Anxiety Disord. 2009;23(1):38-45.

74. Buckner JD, Timpano KR, Zvolensky MJ, Sachs-Ericsson N, Schmidt NB. Implications of comorbid alcohol dependence among individuals with social anxiety disorder. Depress Anxiety. 2008;25(12):1028-1037.

75. Mennin DS, Heimberg RG, Jack MS. Comorbid generalized anxiety disorder in primary social phobia: symptom severity, functional impairment, and treatment response. J Anxiety Disord. 2000;14(4):325-43.
76. Kendall PC, Brady EU, Verduin TL. Comorbidity in childhood anxiety disorders and treatment outcome. J Am Acad Child Adolesc Psychiatry. 2001;40(7):787-94.

77. Nardi AE, Lopes FL, Valenca AM, Freire RC, Nascimento I, Veras AB, Mezzasalma MA, de-Melo-Neto VL, Soares-Filho GL, King AL, Grivet LO, Rassi A, Versiani M. Double-blind comparison of 30 and $60 \mathrm{mg}$ tranylcypromine daily in patients with panic disorder comorbid with social anxiety disorder. Psychiatry Res. 2010;175(3):260-65.

78. Chagas MH, Nardi AE, Manfro GG, Hetem LA, Andrada NC, Levitan MN, Salum GA, Isolan L, Ferrari MC, Crippa JA. [Guidelines of the Brazilian Medical Association for the diagnosis and differential diagnosis of social anxiety disorder]. Rev Bras Psiquiatr. 2010;32(4):444-52.

79. Canadian Psychiatric Association. Clinical practice guidelines.Management of anxiety disorders. Can J Psychiatry. 2006; 51(8 Suppl. 2):9S-91S. 